\title{
Aménagement de Vieux-Pré
}

\author{
The Vieux-Pré dam
}

\author{
S. Alam \\ Neyrpic, Grenoble \\ R. Longuemare \\ EDF, REAL Chambéry
}

\section{Introduction}

L'Aménagement du Vieux-Pré est situé dans les contreforts lorrains du Massif des Vosges dans l'Est de la France.

Le barrage est implanté sur un resserrement de la vallée du ruisseau du Vieux-Pré, sous-affluent de la Meurthe (fig. 1). L'ouvrage est constitué d'une digue en enrochements de grès avec noyau central en argile, de 70 mètres de hauteur, créant un réservoir d'une capacité utile de 50 millions de $\mathrm{m}^{3}$ à la cote 386 de retenue normale.

Cet aménagement non énergétique $\left({ }^{1}\right)$ a pour finalité le soutien du débit d'étiage de la Moselle lorsque celui-ci descend en-dessous d'un seuil fixé à $26 \mathrm{~m}^{3} / \mathrm{s}$, en vue de compenser les débits évaporés dans les aéroréfrigérants de la centrale nucléaire de Cattenom, actuellement en cours de construction à $10 \mathrm{~km}$ à l'aval de Thionville, près de la frontière germano-luxembourgeoise.

En fait, la capacité nécessaire à cette compensation est seulement de $35 \mathrm{hm}^{3}$, mais les possibilités de stockage qu'offrait ce site ont permis d'envisager un projet commun avec l'Agence Financière de Bassin Rhin-Meuse. Cette opération permet à l'Agence de disposer d'une réserve d'eau de $15 \mathrm{hm}^{3}$ pour ses propres besoins, notamment en soutien des étiages de la Meurthe et pour certains besoins agricoles, urbains et industriels dans la région de Nancy-Lunéville.

Le bassin versant propre du réservoir $\left(11 \mathrm{~km}^{2}\right)$ est trop faible pour en garantir son remplissage annuel (apports annuels moyens $6 \mathrm{hm}^{3}$ ). C'est donc par pompage à partir de la Plaine, affluent de la Meurthe, que

(1) Il consomme de l'énergie d'hiver en pompage et la restitue en été par turbinage. cette retenue sera alimentée au moment des hautes eaux d'hiver et de printemps. Un bassin d'accumulation, une station de pompage de $12 \mathrm{MW}$ et une adduction hydraulique souterraine sont donc nécessaires pour assurer cette fonction. En été sec, les débits de soutien d'étiages transiteront en sens inverse par le circuit hydraulique, l'énergie de pompage étant ainsi en partie récupérée par turbinage grâce aux groupes binaires réversibles.

\section{Les ouvrages annexes du barrage}

L'importance de la capacité de la retenue par rapport aux apports gravitaires et les possibilités de laminage offertes par le barrage sont telles que l'on aurait pu se passer d'ouvrage d'évacuation des crues. Cependant, en plus de son caractère de sécurité indispensable, cet ouvrage est davantage un organe permettant, le retour automatique du plan d'eau à sa cote normale après le passage d'une crue. C'est également ce qui explique qu'un modeste ouvrage soit suffisant pour assurer cette fonction.

L'évacuateur, étudié sur modèle réduit, est donc composé d'un déversoir annulaire $\emptyset 4 \mathrm{~m}$, calé à la cote 386,20 , raccordé à un puits vertical de section minimale revêtue $(\phi 2 \mathrm{~m})$ et implanté en rive gauche près du barrage (fig. 2). A la base du puits, une galerie de jonction en $\emptyset 2 \mathrm{~m}$ minimal évacue l'eau vers une section rétrécie située à côté de la vidange de fond (fig. 3). L'écoulement emprunte ensuite la galerie de fuite servant à la vidange de fond. Le débit prévisible à évacuer est de l'ordre de $6,5 \mathrm{~m}^{3} / \mathrm{s}$, et la cote des plus hautes eaux fixée à 386,70 .

Le barrage comporte également une vidange de fond dont le débit maximal a été fixé à $20 \mathrm{~m}^{3} / \mathrm{s}$ compte-tenu des possibilités d'écoulement qu'offre le ruisseau du Vieux-Pré à l'aval du barrage. Lors de l'examen du 
projet par le Comité Technique Permanent des Barrages, ce dernier a demandé une capacité de vidange plus importante pour pouvoir, en cas de nécessité, ramener la poussée hydraulique totale sur le barrage à la moitié de sa valeur dans un délai d'une semaine; un complé- ment de vidange de $30 \mathrm{~m}^{3} / \mathrm{s}$ a donc été prévu en aménageant un piquage blindé à l'extrémité aval de la galerie d'amenée et débouchant directement dans le bassin d'accumulation inférieur de la Plaine par l'intermédiaire d'un robinet à jet creux.
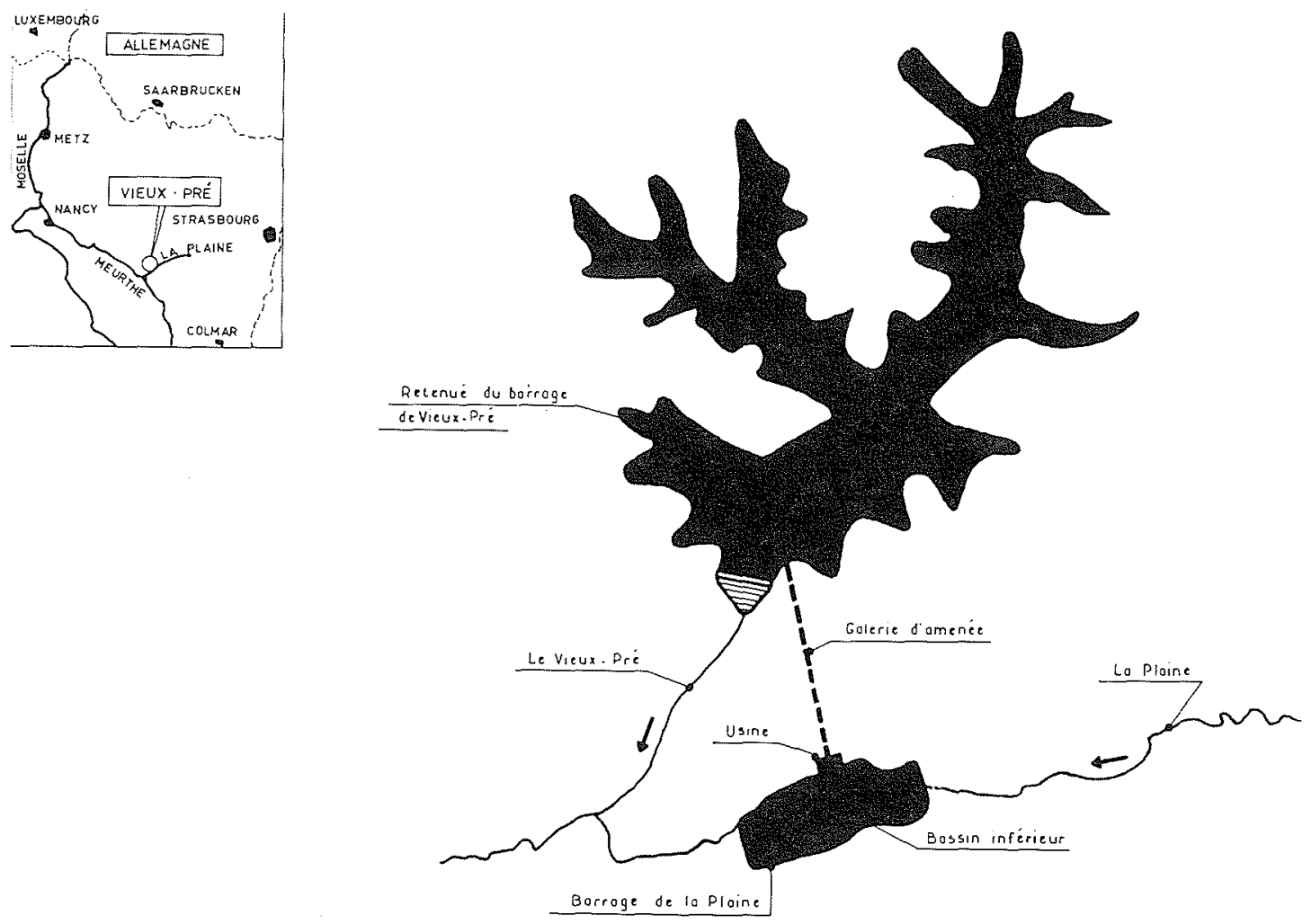

Figure 1

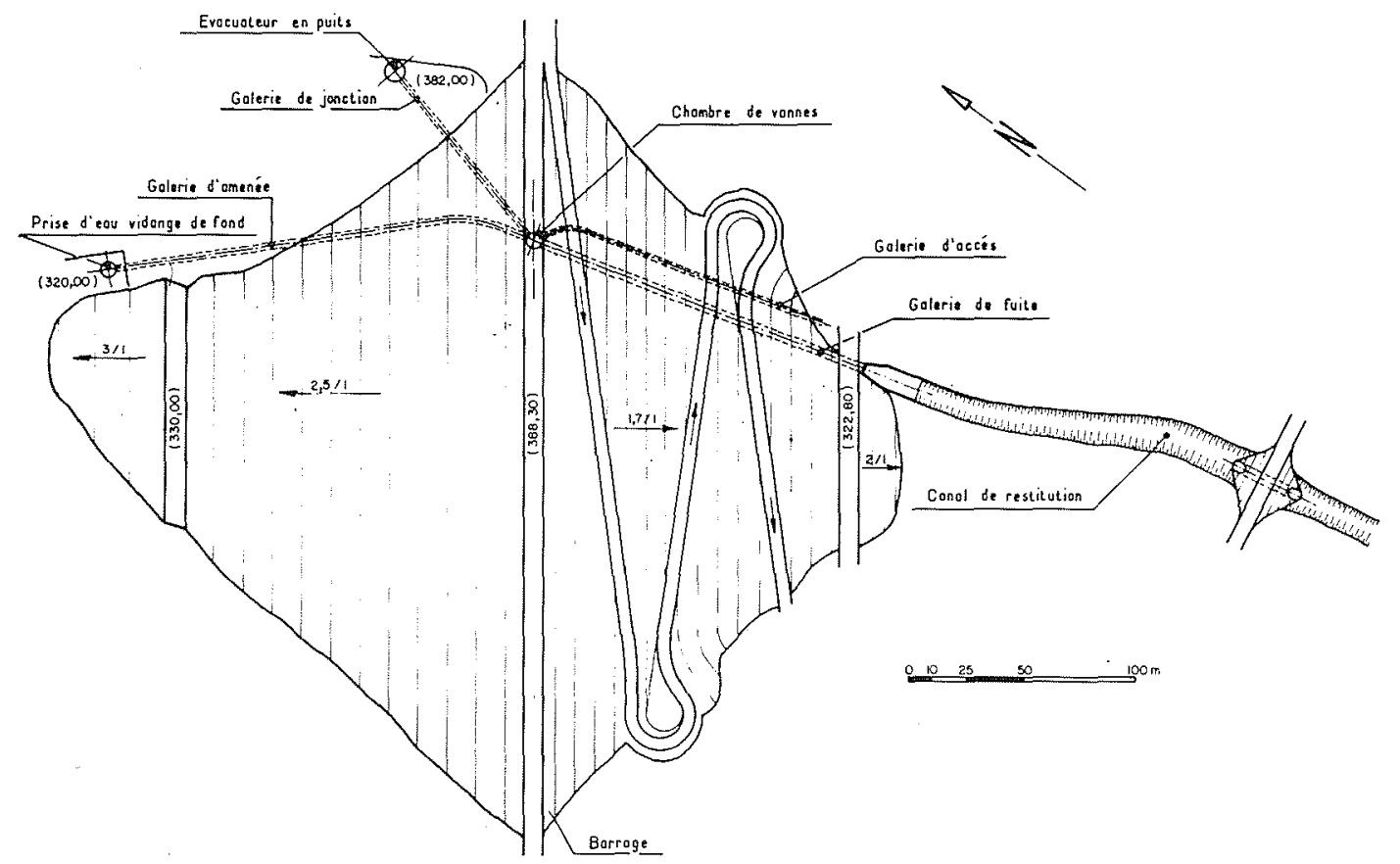

Figure 2 


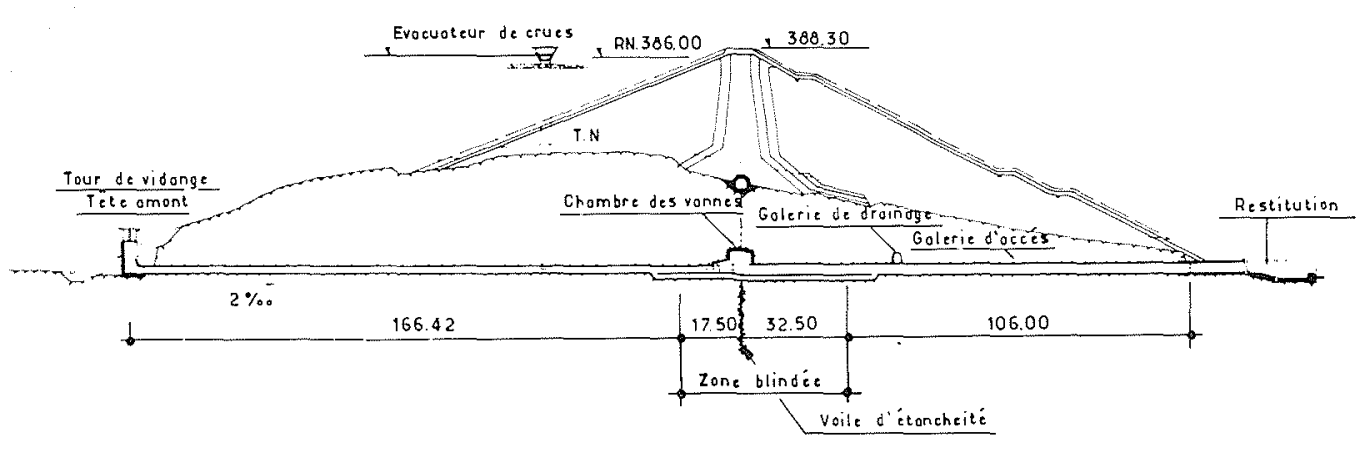

Figure 3

La vidange de fond est aménagée en galerie souterraine sous l'appui rive gauche du barrage. Le circuit comprend un ouvrage d'entonnement classique en tour verticale de $\emptyset 4 \mathrm{~m}$ alimentant une galerie en $\emptyset 2 \mathrm{~m}$ sur environ $190 \mathrm{~m}$ dont les 15 derniers mètres sont blindés à l'amont immédiat de la chambre des vannes, située dans le plan général d'étanchéité du barrage (fig. 2 et 3 ).

Le circuit de vidange de fond est commandé par 2 vannes plates en série comprenant respectivement de l'amont vers l'aval : une vanne de garde $(1,25 \mathrm{~m} \times 1,60 \mathrm{~m})$ et une vanne de réglage $(0,60 \mathrm{~m} \times 1,00 \mathrm{~m})$. L'écoulement emprunte ensuite une galerie de fuite de $150 \mathrm{~m}$ de longueur environ en section fer à cheval $\left(\begin{array}{ll}\phi & 3 \mathrm{~m}\end{array}\right)$, blindée en radier et piédroits sur les 30 premiers mètres. Un décroché du radier permet l'aération du jet à la sortie de la vanne, le débit d'air étant fourni par la galerie d'accès à la chambre des vannes. La restitution de l'écoulement dans le ruisseau du Vieux-Pré s'effectue par l'intermédiaire d'un bassin d'amortissement classique avec décrochement du radier à l'extrémité aval de la galerie de fuite.

\section{Le modèle}

La construction du modèle et les essais ont été confiés à la SOGREAH à Grenoble. Après étude préliminaire, il a été décidé de construire un seul modèle pour les 2 fonctions - évacuateur de crues et vidange de fond - à l'échelle du $1 / 20^{\mathrm{e}}$ avec les limites suivantes :

- une partie de la retenue autour de la tête de l'évacuateur, l'évacuateur complet, le déversoir annulaire, le puits, la galerie de jonction et le débouché dans la chambre des vannes,

- les 20 premiers mètres de la galerie de vidange en amont des vannes, la chambre des vannes avec un morceau de la galerie d'accès, la galerie de fuite, l'ouvrage de dissipation aval et le canal de restitution au ruisseau du Vieux-Pré.

L'alimentation était prise dans la retenue représentée pour l'évacuateur mais par l'intermédiaire d'une vanne permettant de contrôler la charge hydraulique correcte à admettre en amont de la vanne de vidange.

Les ouvrages principaux étaient réalisés en plastique transparent. Outre les appareils de mesures traditionnels (déversoirs, prises de pression, pointes limnimétriques, micro-moulinet, etc...), un système de mesure du débit d'air était installé dans la galerie d'accès. A noter que l'échelle choisie (au $1 / 20^{\mathrm{e}}$ ) peut être considérée comme suffisamment importante pour obtenir une bonne approche de ces phénomènes d'entrânement d'air.

\section{But de l'étude}

L'étude sur modèle réduit des ouvrages annexes du barrage du Vieux-Pré avait pour objet d'examiner: a) le fonctionnement de l'évacuateur de crues:

- entonnement au niveau du seuil annulaire, écoulement dans le puits;

- réception à la base du puits, mise en charge, aération de la veine au départ de la galerie de jonction, possibilité de loger un diaphragme au départ de cette galerie à la base du puits pour assurer un écoulement libre;

- débouché dans la galerie de fuite, aération du jet, zone de l'impact en fonction des débits, balancement de l'écoulement sur le radier.

b) le fonctionnement de la vidange de fond :

- restitution des débits dans la galerie de fuite, vanne de contrôle partiellement ou totalement ouverte, examen des écoulements

- mesure du débit d'air parvenant dans la chambre des vannes,

- examen de conditions d'entraînement en fonction du débit ;

- examen des conditions aval comme pour l'évacuateur.

c) le fonctionnement simultané de la vidange de fond et de l'évacuateur:

- contrôle qualitatif de l'écoulement dans la galerie de fuite.

d) le fonctionnement du bassin de dissipation :

- examen des conditions de dissipation d'énergie dans le bassin prévu à cet effet et des conditions de restitution à la rivière.

\section{Les résultats}

\section{L'évacuateur de crues}

La mise au point de l'évacuateur de crues avec un aussi faible débit à évacuer a été relativement facile. 


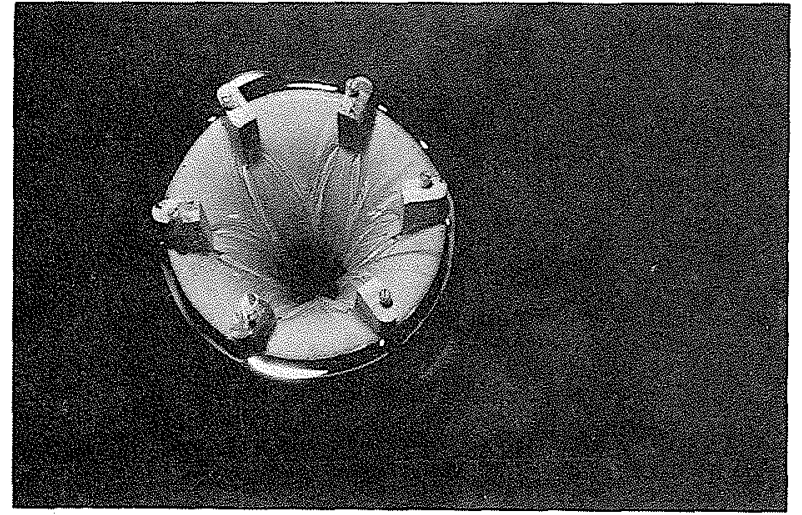

Photo 1

Evacuateur de crues en puits tulipe d'entonnement étudié sur le modèle au $1 / 20^{\circ}$

- Ecoulement sur le seuil annulaire et dans l'entonnement du puits vertical

- Débit évacué : $6,5 \mathrm{~m}^{3} / \mathrm{s}$

Finalement, le seuil déversant annulaire a été réduit à $10 \mathrm{~m}$ au lieu de $17 \mathrm{~m}$ ainsi que le diamètre du puits vertical à $2 \mathrm{~m}$ au lieu de $2,50 \mathrm{~m}$. Le coefficient de débit d'un tel ouvrage est de 0,485 .

Après une chute libre d'environ $60 \mathrm{~m}$, la dissipation d'énergie est acquise grâce à un épais matelas d'eau émulsionnée d'air créé par un surcreusement de la base du puits. Par contre, l'écoulement dans la galerie de jonction n'a pu être amélioré qu'en augmentant sa pente à $5 \%$ pour obtenir un écoulement torrentiel stable et bien aéré et en pinçant très progressivement le conduit au débouché de la chambre des vannes de vidange (fig. 3).

Avec ces ouvrages, il a été possible d'évacuer un débit maximal de $8,5 \mathrm{~m}^{3} / \mathrm{s}$ sans que la galerie de jonction se mette en charge ni que le puits de l'évacuateur soit saturé.

La vitesse de l'eau à la sortie de la galerie de jonction dans la galerie de fuite est faible $(8,5 \mathrm{~m} / \mathrm{s})$, ce qui a permis d'obtenir un écoulement relativement stable dans la galerie de fuite à la vitesse de $2,5 \mathrm{~m} / \mathrm{s}$ au moment de sa restitution dans le bassin aval.

\section{La vidange de fond}

C'est l'ouvrage délicat à mettre au point compte-tenu de l'importance des vitesses de l'écoulement à la sortie de la vanne: de l'ordre de $35 \mathrm{~m} / \mathrm{s}$ pour une charge brute maximale de $66 \mathrm{~m}$ à la cote de retenue normale du barrage.

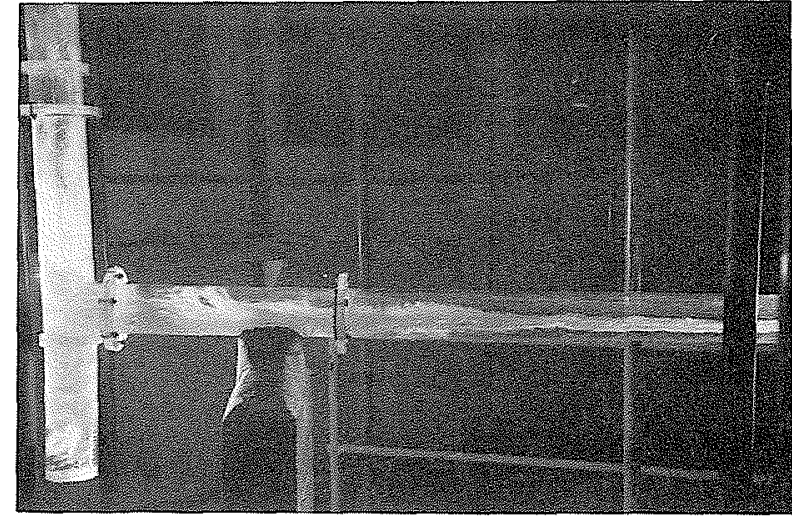

Photo 2

Puits vertical et galerie de jonction

- Conditions d'écoulement à la base du puits et au départ de la galerie de jonction

- Débit évacué : $6,5 \mathrm{~m}^{3} / \mathrm{s}$

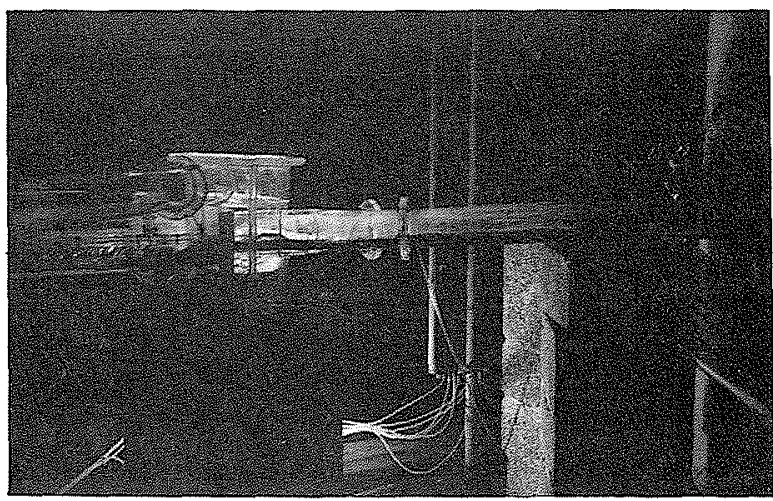

Photo 3

Galerie de jonction et chambre des vannes

- Ecoulement dans la partie aval de la galerie de jonction et au départ de la galerie de fuite

- Evacuation de $6,5 \mathrm{~m}^{3} / \mathrm{s}$

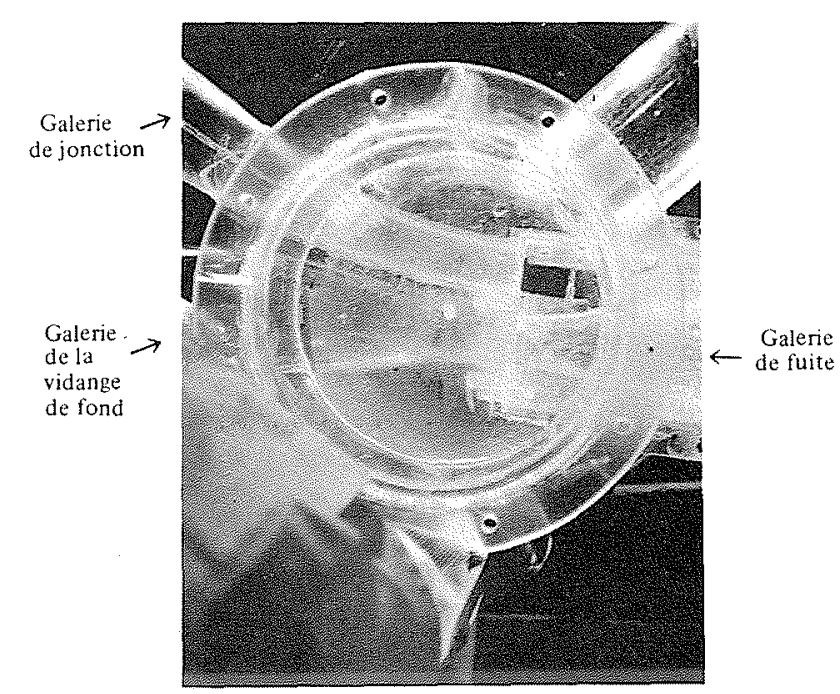


Pour résoudre ce problème, nous nous sommes inspirés de nos études de la vidange de fond du barrage de Grand'Maison dont les conditions de fonctionnement étaient encore plus sévères: débit $100 \mathrm{~m}^{3} / \mathrm{s}$, vitesse de l'ordre de $50 \mathrm{~m} / \mathrm{s}$ pour une charge brute maximale de $140 \mathrm{~m}$.

Les écoulements à grande vitesse sont le siège de phénomènes de cavitation qui provoquent des dégradations inacceptables aux ouvrages de génie civil. La cavitation est engendrée par les défauts de surface des parois qu'il est souvent difficile d'éliminer à l'exécution. De plus, dans le cas d'une vanne plate, les rainures de la vanne sont très sensibles à ce phénomène.

La présence d'air inclus au sein de l'écoulement rapide en contact avec une paroi constitue une émulsion air - eau qui s'oppose efficacement à la formation de la cavitation.

Le principe de l'aération d'une veine liquide à grande vitesse est simple: il convient d'entrainer le maximum de débit d'air par frottement de la veine liquide sur l'air. Pour cela, le procédé classique consiste à aérer la veine sur ses quatre faces par un élargissement brutal des bajoyers et un décrochement brusque du radier à l'aval de la vanne, ces deux décrochements étant, bien entendu, correctement alimentés en air.

Mais le problème est de maintenir cette aération en radier tant qu'il y a risque de cavitation. La solution consiste à réintroduire une nouvelle aération par un second décrochement aval si les vitesses de l'écoulement sont encore relativement importantes. C'est le cas de la vidange de Grand'Maison où un deuxième décrochement est réalisé à l'aval de la partie blindée. Les essais ont montré que ce n'était pas nécessaire pour la vidange du Vieux-Pré.

En plus de cette aération et par sécurité, la galerie de fuite à l'aval de la vanne sera blindée sur une longueur suffisante, l'acier résistant mieux que le béton à la cavitation. Toutefois, il devra être correctement ancré au béton pour tenir aux sous-pressions éventuelles.

Les premiers essais ont indiqué que le dispositif de guidage placé à l'aval de la vanne et parallèlement à l'axe du pertuis permettait de bien centrer le jet dans la galerie de fuite pour toutes les ouvertures de la vanne. Cependant, l'absence d'aération des rainures aux ouvertures partielles présentait un certain risque de cavitation. Pour cette raison, le dispositif de guidage a été supprimé et le bord aval de la rainure décalé de $40 \mathrm{~mm}$.

Avec cette disposition, l'écoulement se détache bien des rainures qui restent complètement hors d'eau. Cependant, en l'absence du dispositif de guidage, le jet s'élargit et plonge plus rapidement provoquant un impact sur le radier avec une dispersion très violente et inacceptable.

Par ailleurs, à ouverture partielle de la vanne, le jet s'aplatit et pénètre dans les rainures. Ce phénomène est d'autant plus marqué que la vanne est fermée. Cette

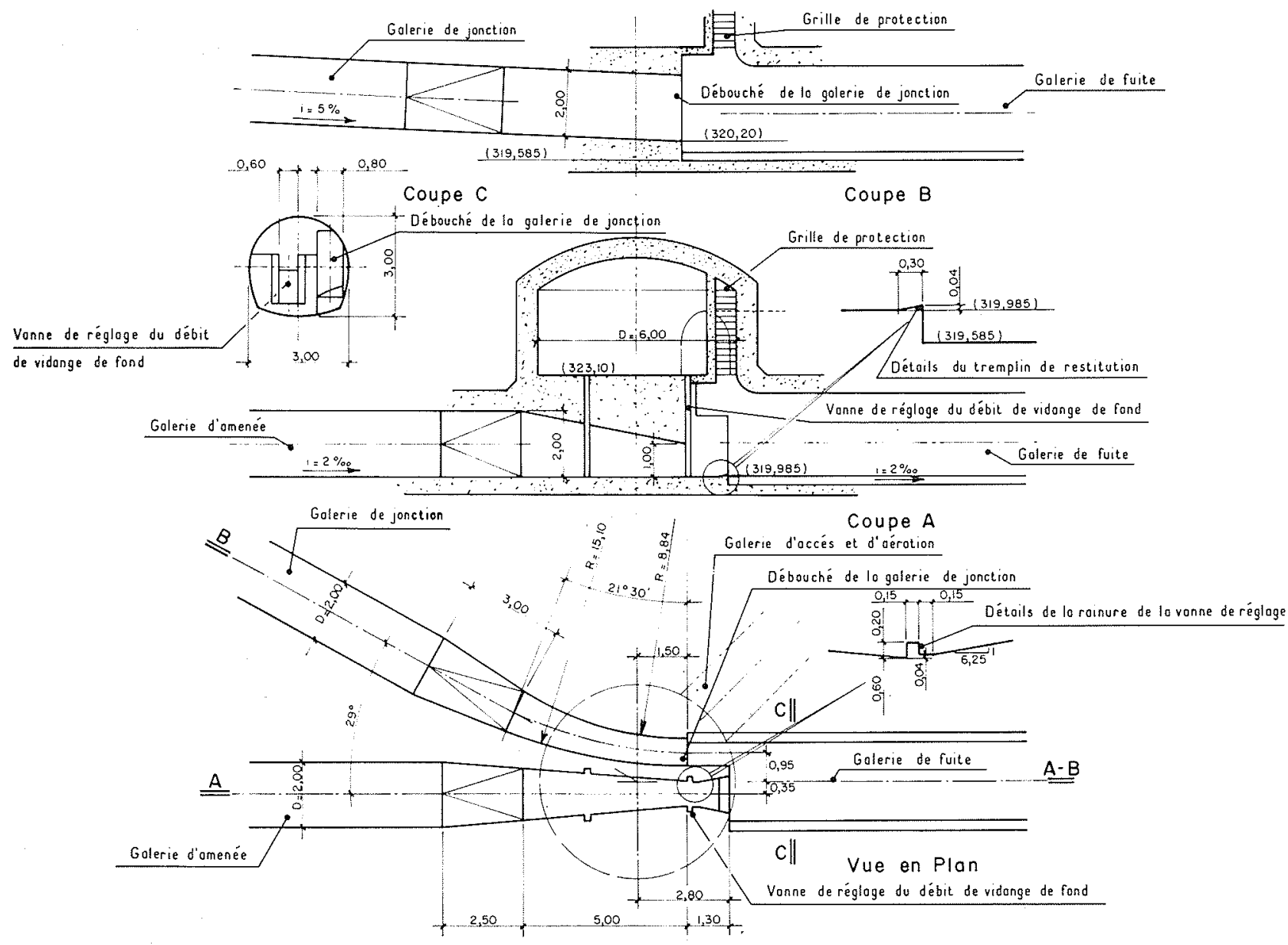

Figure 4 
disposition entraine un impact du jet sur le radier et les parois de galerie avec des projections très instables jusqu'au sommet de la voûte.

Pour pallier ce défaut, différentes sortes d'onglets déflecteurs ont été essayés à l'amont du plan de la vanne. Les onglets verticaux augmentent quelque peu la contraction latérale du jet mais il n'a pas été possible de dénoyer les rainures pour toutes les ouvertures de la vanne.

Un onglet horizontal placé sous le seuil de la vanne a permis, en relevant le jet, de reporter à l'aval son impact sur le radier à l'ouverture totale mais, à ouvertures partielles, le jet est toujours projeté contre la voûte de la galerie.

Les essais précédents ont montré qu'il était nécessaire de revenir au système de guideaux pour orienter le jet de telle sorte que son impact sur le radier et sur les parois de la galerie se fasse tangentiellement et sans perturbation.

Le système représenté sur la figure 4 qui a été mis au point et comprend :

- le décalagé de l'arête aval de la rainure,

- un système de guidage divergent,

- et un onglet en radier.

La figure 5 représente les différentes trajectoires du jet pour quatre ouvertures de la vanne correspondant aux débits de $5,10,15$ et $20 \mathrm{~m}^{3} / \mathrm{s}$.
On a observé que la grande vitesse et la nature de l'écoulement à la sortie de la vanne établissent un régime super-critique sur toute la longueur de la galerie de fuite pratiquement à partir d'un débit de l'ordre de 2 à $3 \mathrm{~m}^{3} / \mathrm{s}$.

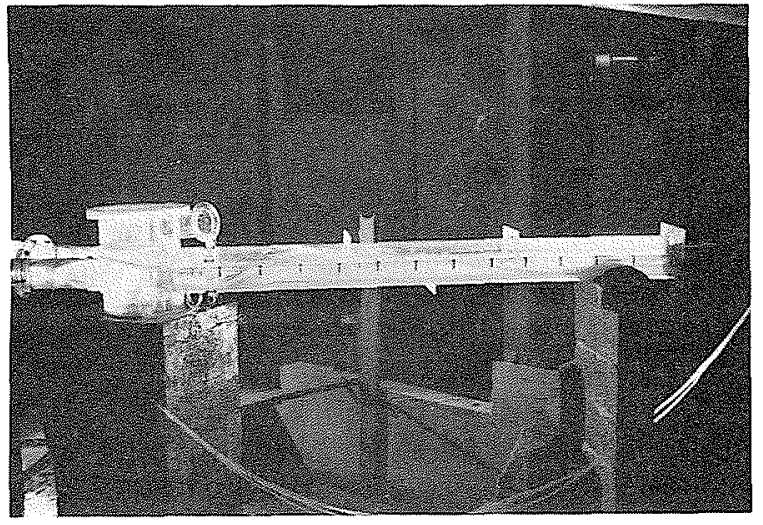

Photo 5

Chambre des vannes et galerie de fuite

Aspect de la dispersion violente du jet dans la galerie de fuite

- vanne avec les bords aval des rainures décalés, sans dispositif de guidage (impact du jet sur le radier de la galerie de fuite) - débit de vidange de fond : $20 \mathrm{~m}^{3} / \mathrm{s}$

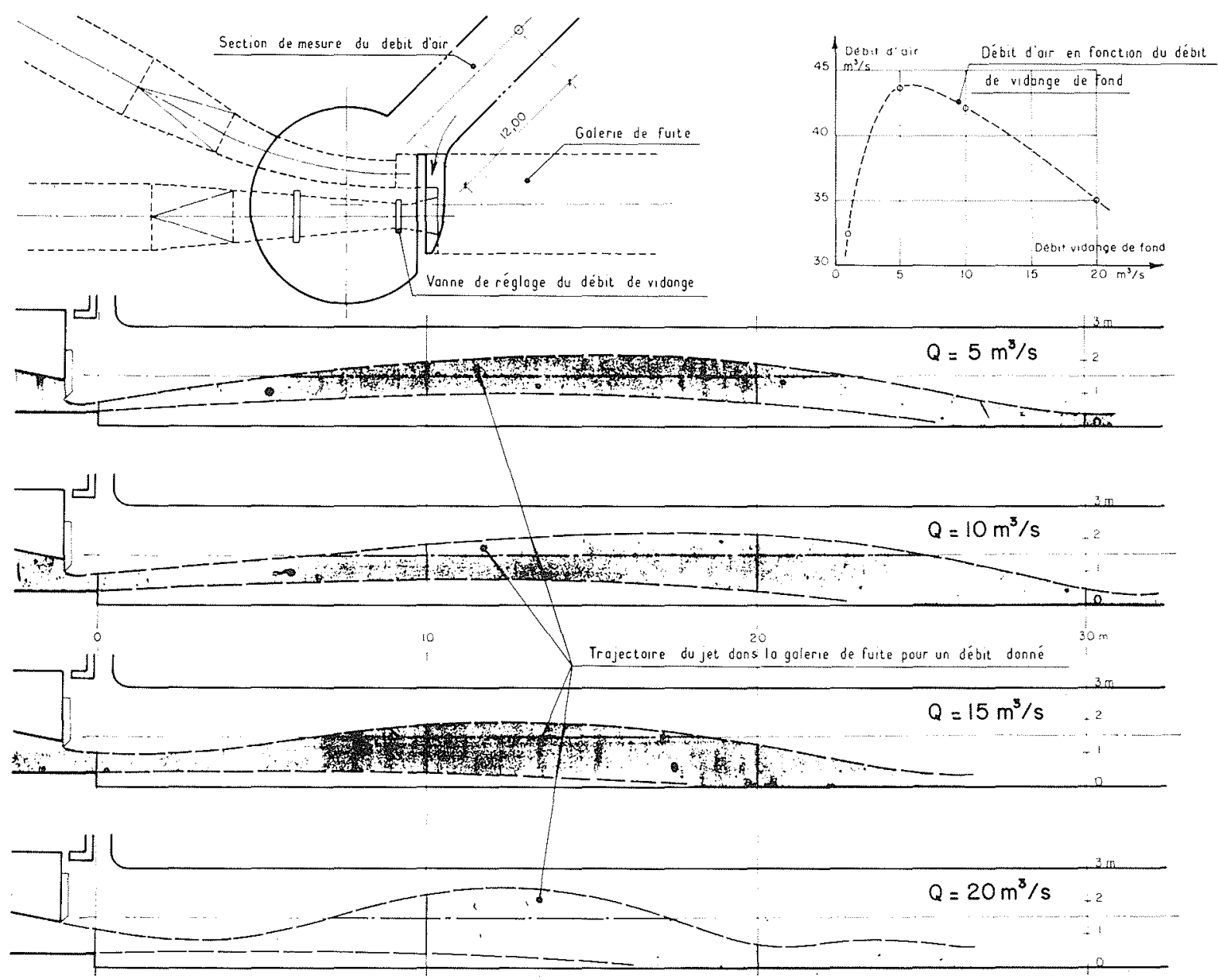

Figure 5 
L'écoulement se fait sans balancement d'ondes de choc gênantes.

L'écoulement simultané de l'évacuateur et de la vidange qui, en principe, a une probabilité infime de se produire, tout au moins pendant une durée importante, s'effectue convenablement dans la galerie de fuite.

On constate que la dissipation d'énergie s'effectue assez rapidement. En effet, la vitesse de $35 \mathrm{~m} / \mathrm{s}$ à la sortie de la vanne tombe à $20 \mathrm{~m} / \mathrm{s}$ à $50 \mathrm{~m}$ à l'aval, et

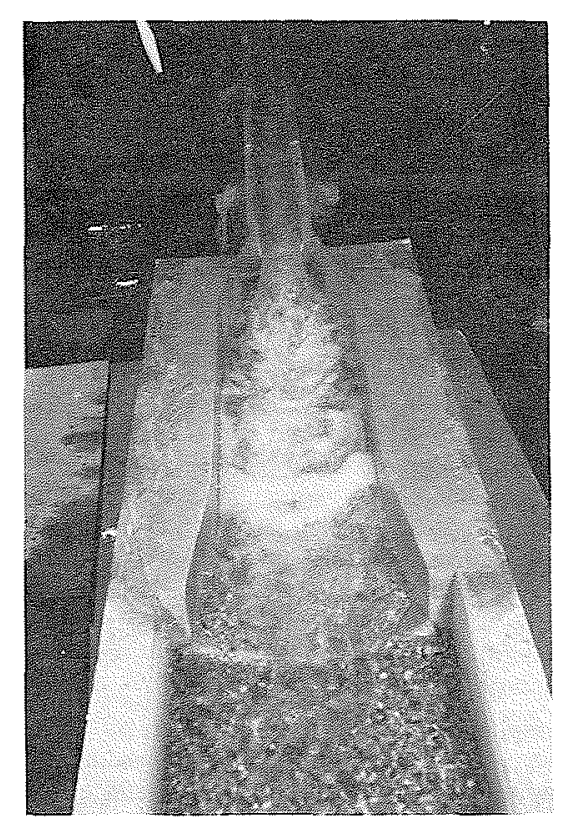

Photo 6

Bassin de dissipation

Aspects de la dissipation et de l'écoulement à l'aval du bassin pour un débit de $20 \mathrm{~m}^{3} / \mathrm{s}$ par la vidange de fond n'est plus que de $15 \mathrm{~m} / \mathrm{s}$ à $150 \mathrm{~m}$, c'est-à-đire à la restitution de la galerie dans un bassin de tranquillisation très classique à la sortie duquel la vitesse de l'écoulement n'est plus que de l'ordre de $3,5 \mathrm{~m} / \mathrm{s}$. Le débit d'air entrainé par l'écoulement de la vidange a été mesuré sur le modèle à l'aide d'un anémomètre placé dans la galerie d'accès

Sur la figure 5 est indiquée la courbe du débit d'air en fonction du débit de la vidange. Nous avons constaté que l'entraînement d'air dépend essentiellement de la forme du jet. Il est d'autant plus important que l'interface eau - air est le plus grand, ce qui se produit à ouverture très partielle de la vanne pour un débit de l'ordre de $5 \mathrm{~m}^{3} / \mathrm{s}$.

Bien que les conditions de similitude d'entraînement d'air soient mal connues, il est remarquable de constater que les débits d'air mesurés sur le modèle sont du même ordre de grandeur que ceux que l'on peut calculer à partir des abaques publiés par Hari R. Sharma (Proceeding of ASCE 1976) pour des conditions comparables.

Il faut cependant noter que la répartition du débit d'air entre la galerie d'accès à la chambre des vannes et le puits de l'évacuateur mesurée sur le modèle n'est pas représentative du fait que la galerie d'accès est très courte sur le modèle. Il est néanmoins possible d'estimer que sur le prototype la répartition du débit d'air sera approximativement équilibrée entre les 2 ouvrages.

\section{Conclusion}

L'étude sur modèle réduit des ouvrages annexes du barrage du Vieux-Pré a permis essentiellement de mettre au point des dispositifs très particuliers d'aération de l'écoulement à grande vitesse à la sortie de la vanne pour pallier les risques de cavitation et assurer la pérennité des ouvrages de génie civil.
M. le Président s'interroge sur la limite inférieure du domaine des grandes vitesses $(15,20$ ou $30 \mathrm{~m} / \mathrm{s}$ ?) puis présente les auteurs du rapport.

M. LONGUEMARE expose la communication, accompagnée de diapositives.

A la demande du Président, M. Longuemare précise que le modèle dont M. ALAM a eu la responsabilité de la fabrication et de l'exploitation - n'existe plus; avant la mise au point définitive des formes hydrauliques, plusieurs variantes ont été envisagées et étudiées : pour l'évacuateur, c'est un puits vertical qui a été retenu. C'était la solution la plus facile et la plus économique à réaliser (percement au raise-drill dans les grès des Vosges)

$M$. le Président rappelle que le modèle réduit est une démonstration très claire de l'application pragmatique d'un problème dont les données sont bien connues mais dont les solutions échappent pratiquement au calcul ; pour le moment, c'est finalement le modèle réduit qui peut donner une solution suffisamment précise et correcte à un décollement de veine ou à une zone d'impact. 
Vu les difficultés rencontrées par les instabilités hydrauliques créées par les larges rainures $(15 \mathrm{~cm})$ de la vanne de réglage (Fig. 4), M. BEZINGE demande pourquoi EDF ne procède-t-il pas à une réduction importante de ces rainures? A Grande Dixence, en effet, pour des vannes de dimensions semblables, la largeur des rainures n'est que de $4,5 \mathrm{~cm}$, avec construction adéquate du tablier, pour éviter des usures importantes (eaux très chargées) et les résultats sont excellents. Les formes des courbes d'entrainement d'air (appelées "moustaches") sont liées également à la forme amont du tablier des vannes planes : sur la vidange de fond du barrage de Grande Dixence (vannes planes de $0,40 \mathrm{~m} \times 0,60 \mathrm{~m}$, pression maximale $290 \mathrm{~m} \mathrm{CE}$ ), des mesures d'absorption d'air, avec $120 \mathrm{~m}$ de chute, effectuées en 1957 , ont donné des rapports de débit eau/air de 1 à 16 , au maximum sous $20 \%$ d'ouverture. Ce phénomène met en dépression les $30 \mathrm{~km}$ de puits et de galerie du barrage en quelques minutes. A l'époque, ces résultats ne recoupaient pas les valeurs de l'U.S. Army. Les pertes de charge sur l'amenée de l'air doivent certainement jouer un grand rôle sur ce rapport; la dépression mesurée au départ du jet était d'environ $50 \mathrm{~m} \mathrm{CE}$, ce qui est élevé. La courbe de consommation d'air est de forme semblable à celle donnée par la figure 5 .

En ce qui concerne la forme de l'arête du couteau de la vanne, M. LONGUEMARE précise qu'il y a deux théories : soit le couteau de la vanne avec contraction maximale (vanne à tendance à la fermeture), soit la vanne inversée. Mais de toute façon, quelle que soit la forme choisie pour le couteau, il est toujours possible (comme cela avait été expliqué par une diapositive) avec un décroché du radier et avec un petit déflecteur amont (appelé "onglet") de modeler le jet de façon à avoir le maximum d'aération sur les 4 faces et surtout d'avoir un jet stable.

M. ANDRE indique que, d'après les essais effectués depuis 20 ans environ sur des installations en service, on peut déduire les constatations suivantes

- les débits d'air on tété plus grands que ceux prévus.

- les lois du débit d'air en fonction du débit d'eau étaient voisines de celles indiquées par M. LONGUEMARE (coefficient d'entrée d'air maximum pour le quart du débit d'eau) ;

- les coefficients (débit d'air/débit d'eau) varient beaucoup :

Serre-Ponçon : de 0,6 à 1 ,

Saint-Cassier : de 1,7 à 6 ,

Sautet : de 3 à 10 ,

MM. LONGUEMARE et ALAM rappellent que l'échelle du modèle joue énormément, pour ces problèmes d'entrainement d'air, pour la transposition des phénomènes du modèle au prototype. Pour Serre-Ponçon, l'échelle du modèle était au $1 / 50^{\mathrm{e}}$ et la reproduction était donc đifficile; pour Vieux-Pré, les chiffres cités devraient être plus justes, d'autant qu'on sait mieux maintenant interprêter les débits d'air. Le choix de l'échelle du modèle doit donc être effectué avec beaucoup d'attention et il faut être très prudent pour la conception des reniflards et des galeries d'aération.

A la demande de M. THIRRIOT, il est précisé que le surcreusement du fond du puits - qui avait été estimé à $2 \mathrm{~m}$ - a été porté, après essai sur modèle et pour avoir un meilleur amortissement, à $4 \mathrm{~m}$.

Aux demandes de $M$. le Président, il est confirmé que le début de la mise en eau de Vieux-Pré est aujourd'hui prévu pour 1984 (actuellement le décapage des appuis du barrage est réalisé, les galeries sont en cours d'exécution et/ou de bétonnage) et que le fonctionnement simultané des 2 ouvrages (évacuateur de surface et vidange de fond) devrait être très exceptionnel (puisqu'il faudrait en effet la conjonction d'une grosse crue et d'une défaillance du barrage).

Pour ce qui concerne les blindages, M. POST rappelle qu'au barrage de Tarbela, au Pakistan, l'arrachement du blindage du radier d'une vidange de fond fonctionnant sous des vitesses de $45 \mathrm{~m} / \mathrm{s}$, a été expliqué par un phénomène de vibrations. Ces vibrations, dues aux pulsations dynamiques de l'écoulement combinées à une insuffisance des raidisseurs et ancrages, auraient provoqué une rupture des soudures de la tôle et son arrachement par l'introduction d'une partie du terme de vitesse et non pas par un phénomène classique de sous-pression.

M. LONGUEMARE rappelle un cas intéressant, celui de la vidange de fond du barrage de Grand'Maison. Celle-ci est implantée parallèlement à la galerie de dérivation provisoire qui continue d'alimenter la vieille usine du Riviers qui sera court-circuitée par le nouvel aménagement. L'intérêt de cette disposition est double. En premier lieu, elle apporte une grande souplesse dans le planning de montage des vannes de vidange. En second lieu, lorsque la dérivation provisoire est bouchée, en situation définitive, sa proximité permet de l'utiliser comme galerie d'aération de la galerie de vidange.

En complément, M. POST indique qu'au barrage de SerrePonçon, le calcul de l'aération des vidanges de fond fut l'occasion de nombreuses discussions. Finalement, c'est la prudence qui l'emporta. Le conduit d'aération qui sert aussi de galerie d'accès pour le montage des vannes est de très grandes dimensions (section de l'ordre de $18 \mathrm{~m}^{2}$ ). On y a mesuré malgré tout, lors des essais des vidanges sous la pleine charge $130 \mathrm{~m}$, des vitesses d'air dépassant $100 \mathrm{~km} /$ heure. Si l'on a mesuré un débit d'air inférieur au débit d'eau pour le débit total des deux vidanges fonctionnant simultanément, et aérées par la même galerie, par contre, lors du fonctionnement d'une seule vidange, on a mesuré un débit d'air à peu près égal au débit d'eau (c'està-dire environ $600 \mathrm{~m}^{3} / \mathrm{s}$ ).

Les questions étant épuisées, M. le Président remercie à nouveau MM. LONGUEMARE et ALAM pour leur très intéressante communication ainsi que tous les intervenants au débat. 